Part of Journal of Research of the National Bureau of Standards, Volume 27, November 1941

\title{
STRESSES IN A RECTANGULAR KNEE OF A RIGID FRAME
}

\section{By William R. Osgood}

In Research Paper RP1130 ${ }^{1}$ of the National Bureau of Standards a theoretical analysis has been presented of the stresses in a rectangular knee of a rigid frame. This analysis is not free from objection because in the limit, as the cross-sectional areas of the flanges approach zero, a

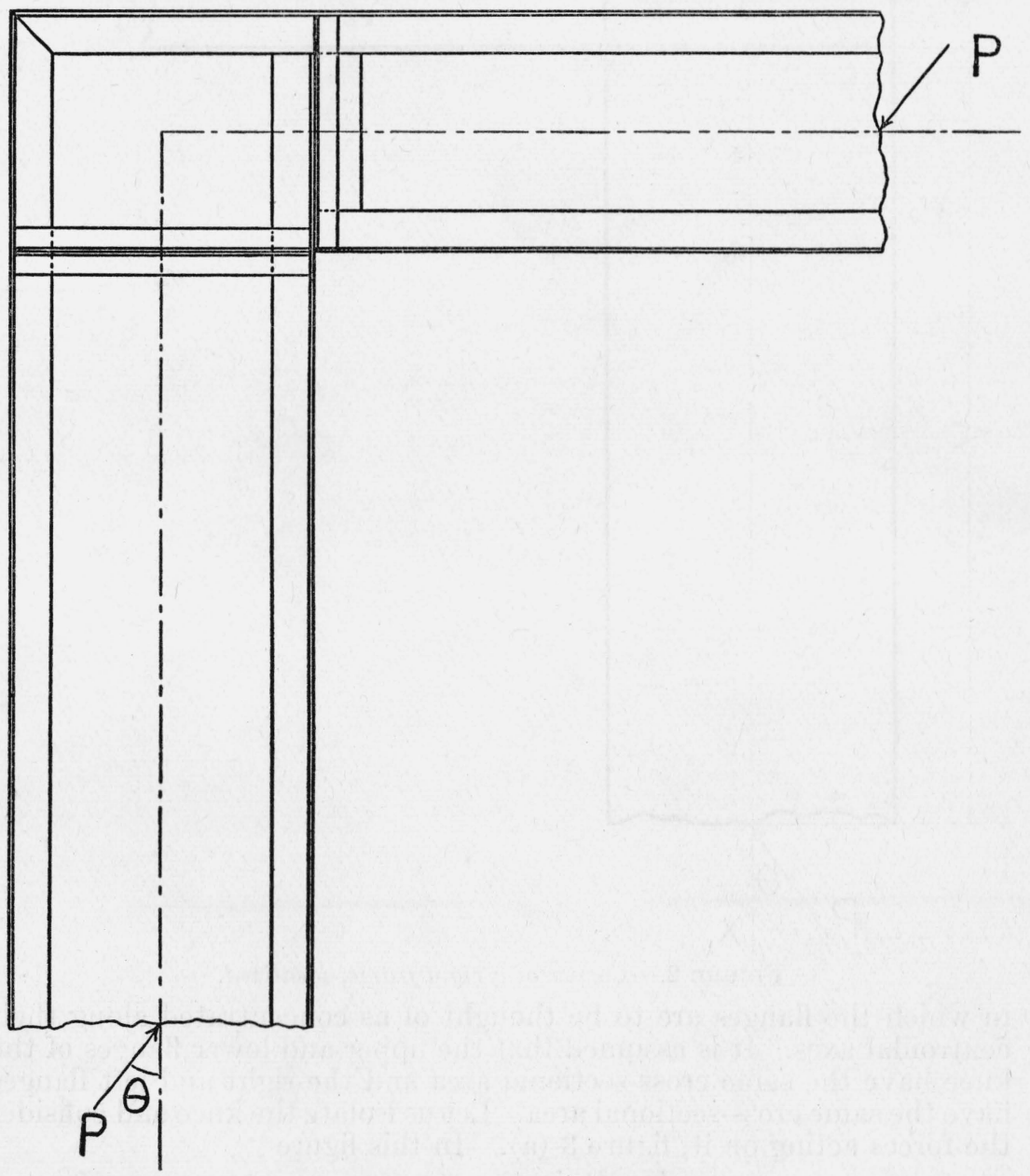

FigURE 1.-Corner:of a rigid frame.

1 J. Research NBS_21, 269 (1938)_RP1130. 
distribution of stress at the boundaries of the knee is approached which departs widely from what appears reasonable. In the present paper an analysis that is largely free from this objection is presented for a knee with two planes of symmetry.

Figure 1 shows in outline a corner of a rigid frame having a rectangular knee, and figure 2 shows an idealized conception of a frame

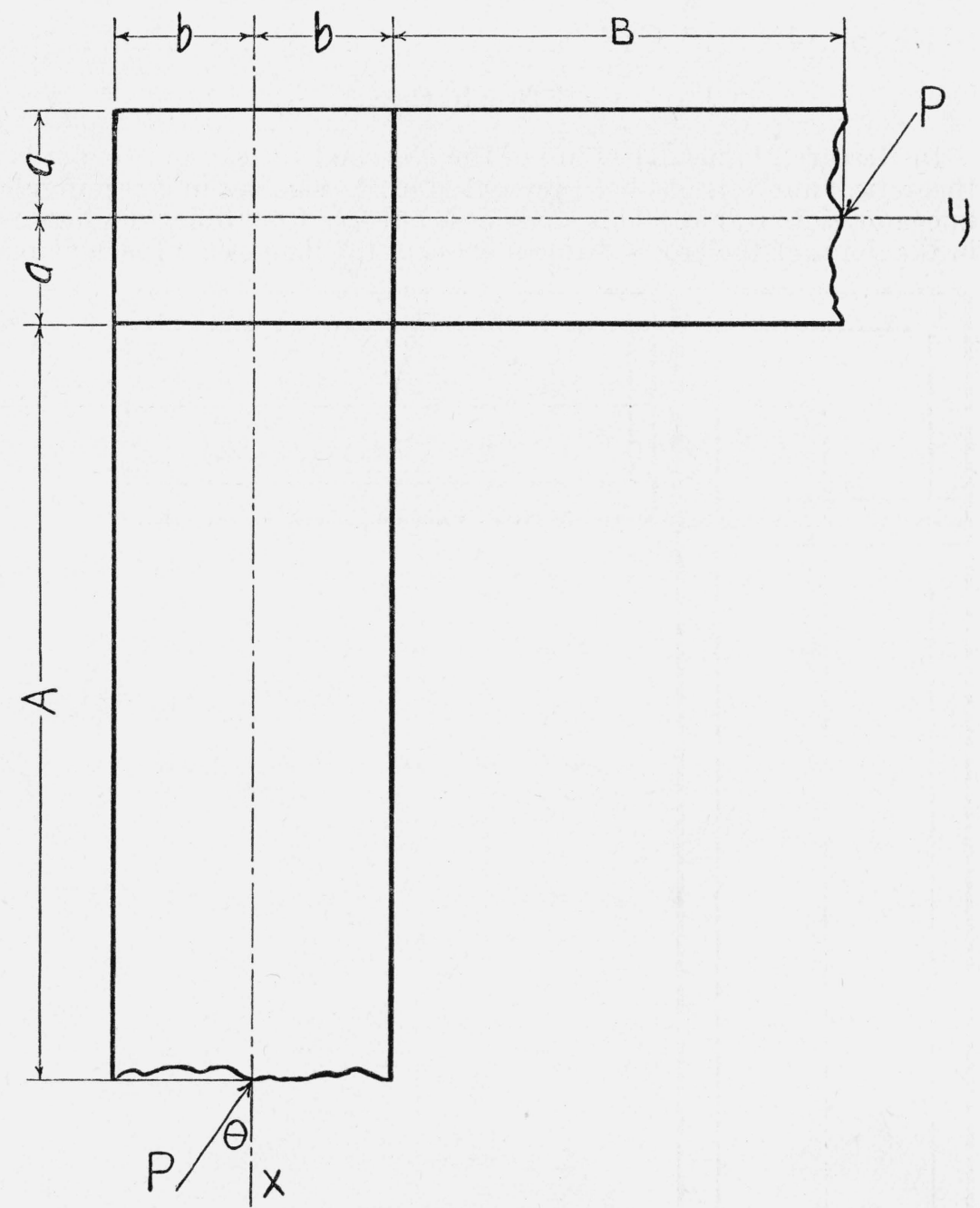

Figure 2.-Corner of a rigid frame, idealized.

in which the flanges are to be thought of as concentrated along their centroidal axes. It is assumed that the upper and lower flanges of the knee have the same cross-sectional area and the right and left flanges have the same cross-sectional area. Let us isolate the knee and consider the forces acting on it, figure 3 (a). In this figure

$$
\begin{aligned}
H & =P \sin \theta, \\
V & =P \cos \theta, \\
M_{0} & =H(A+a)=V(B+b) .
\end{aligned}
$$


where $P, A, B, a, b$, and $\theta$ are the known quantities shown in figure 2 . The system of forces shown in figure 3 (a) may be resolved into the three systems, each in equilibrium, shown in figure 3 (b), (c), and (d). If it is borne in mind that the forces $H$ and $V$ and the couples $H a$, $V b$, and $M_{0}$ are transmitted to the knee by the legs, in which the distribution of normal stress on a cross section is linear, except possibly in the immediate neighborhood of the knee, then it is reasonable to assume that the normal forces $H$ and $V$ are the resultants of uniformly distributed forces, and $H a, V b$, and $M_{0}$ the resultants of approximately uniformly varying forces. Under these assumptions, solutions may be obtained that satisfy the equations of equilibrium and the conditions of compatibility for the three cases of figure 3 (b), (c), and (d). In these solutions, which follow, the origin of coordinates is taken at the center of the knee and the coordinate axes parallel to the sides, as shown in figure $3, t$ is the thickness of the web plate, $A_{a}$ and $A_{b}$ are the areas of

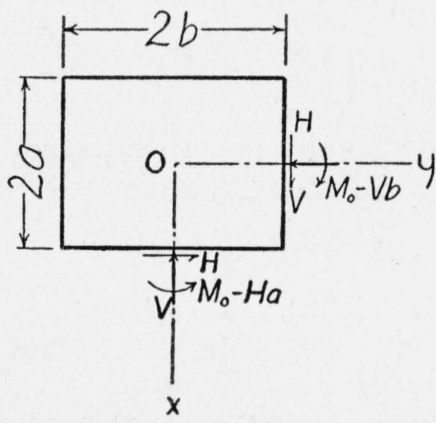

(a)

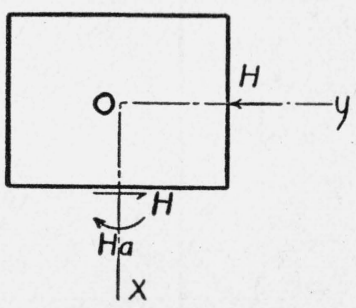

(b)

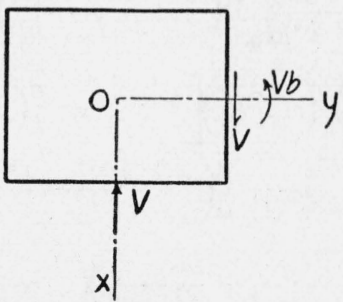

(c)

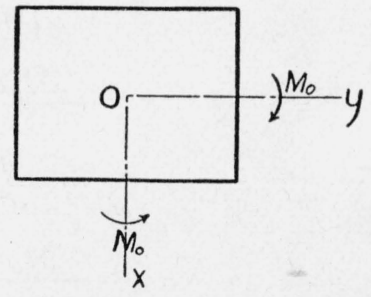

(d)

FIGURE 3.-Loads on knee of rigid frame.

cross sections parallel to the axes of $x$ and $y$, respectively, and $I_{a}$ and $I_{b}$ are the least moments of inertia of $A_{a}$ and $A_{b}$, respectively, with respect to axes perpendicular to the web plate.

For the case of figure 3 (b)

$$
\left.\begin{array}{rl}
\sigma_{x} & =\frac{H a y}{I_{b}}\left[\frac{1}{2}\left(1+\frac{x}{a}\right)-\frac{a t}{2 A_{a}}\left(1-\frac{x^{2}}{a^{2}}\right)+\frac{b^{2} t}{3 a A_{a}} \cdot\left(1-\frac{4 b^{3} t}{15 I_{b}}-\frac{y^{2}}{b^{2}}\right)\right], \\
\sigma_{y} & =-\frac{H}{2 A_{a}}\left(1+\frac{y}{b}\right)\left[1+\frac{b^{3} t}{3 I_{b}} \frac{y}{b}\left(1-\frac{y}{b}\right)\right], \\
\tau_{x y} & =\frac{H a b^{2} t}{2 A_{a} I_{b}}\left(\frac{A_{a}}{2 a t}+\frac{x}{a}\right)\left(\frac{1}{3}+\frac{I_{b}}{b^{3} t}-\frac{y^{2}}{b^{2}}\right) .
\end{array}\right\}
$$


This solution satisfies the following boundary conditions:

$$
\begin{aligned}
& y=-b: \sigma_{y}=0 ; \\
& x=-a: \int_{A_{b}} \sigma_{x} d A=0 ; \\
& x=-a: \int_{A_{b}} \sigma_{x} y d A=0 .
\end{aligned}
$$

The solution for the case of figure 3 (c) is obtained from the preceding solution by replacing $H$ by $V$ and interchanging $a$ and $b$ and $x$ and $y$ :

$$
\begin{aligned}
\sigma_{x} & =-\frac{V}{2 A_{b}}\left(1+\frac{x}{a}\right)\left[1+\frac{a^{3} t}{3 I_{a}} \frac{x}{a}\left(1-\frac{x}{a}\right)\right] \\
\sigma_{y} & =\frac{V b x}{I_{a}}\left[\frac{1}{2}\left(1+\frac{y}{b}\right)-\frac{b t}{2 A_{b}}\left(1-\frac{y^{2}}{b^{2}}\right)+\frac{a^{2} t}{3 b A_{b}}\left(1-\frac{4 a^{3} t}{15 I_{a}}-\frac{x^{2}}{a^{2}}\right)\right], \\
\tau_{x y} & =\frac{V a^{2} b t}{2 A_{b} I_{a}}\left(\frac{A_{b}}{2 b t}+\frac{y}{b}\right)\left(\frac{1}{3}+\frac{I_{a}}{a^{3} t}-\frac{x^{2}}{a^{2}}\right) .
\end{aligned}
$$

The following boundary conditions are satisfied:

$$
\begin{array}{ll}
x=-a: & \sigma_{x}=0 ; \\
y=-b: & \int_{A_{a}} \sigma_{y} d A=0 ; \\
y=-b: & \int_{A_{a}} \sigma_{y} x d A=0 .
\end{array}
$$

For the case of figure $3(\mathrm{~d})$

$$
\begin{aligned}
\sigma_{x}= & -\frac{M_{0} y}{I_{b}}\left\{\frac{1}{2}\left(1+\frac{x}{a}\right)\left[1+\frac{1}{3} \frac{a^{3} t}{I_{a}} \frac{x}{a}\left(1-\frac{x}{a}\right)\right]\right. \\
& \left.-\frac{a^{5} b^{2} t}{3\left(a^{4}+b^{4}\right) I_{a}} \frac{x}{a}\left(1-\frac{4 b^{3} t}{15 I_{b}}-\frac{y^{2}}{b^{2}}\right)\right\}, \\
\sigma_{y}= & -\frac{M_{0} x}{I_{a}}\left\{\frac{1}{2}\left(1+\frac{y}{b}\right)\left[1+\frac{1}{3} \frac{b^{3} t}{I_{b}} \frac{y}{b}\left(1-\frac{y}{b}\right)\right]\right. \\
& \left.-\frac{a^{2} b^{5} t}{3\left(a^{4}+b^{4}\right) I_{b}} \frac{y}{b}\left(1-\frac{4 a^{3} t}{15 I_{a}}-\frac{x^{2}}{a^{2}}\right)\right\}, \\
\tau_{x y}= & -\frac{M_{0} a^{2} b^{2} t}{4 I_{a} I_{b}}\left\{\left(\frac{1}{3}+\frac{I_{a}}{a^{3} t}-\frac{x^{2}}{a^{2}}\right)\left(\frac{1}{3}+\frac{I_{b}}{b^{3} t}-\frac{y^{2}}{b^{2}}\right)\right. \\
& -\frac{a^{2} b^{2}}{3\left(a^{4}+b^{4}\right)}\left[\left(1-\frac{x^{2}}{a^{2}}\right)^{2}-\frac{8 a^{3} t}{15 I_{a}}\left(\frac{1}{3}+\frac{I_{a}}{a^{3} t}-\frac{x^{2}}{a^{2}}\right)\right. \\
& \left.\left.+\left(1-\frac{y^{2}}{b^{2}}\right)^{2}-\frac{8 b^{3} t}{15 I_{b}}\left(\frac{1}{3}+\frac{I_{b}}{b^{3} t}-\frac{y^{2}}{b^{2}}\right)\right]\right\} .
\end{aligned}
$$

This solution satisfies the following boundary conditions:

$$
\begin{array}{ll}
x=-a: & \int_{A_{b}} \sigma_{x} d A=0 ; \\
x=-a: & \int_{A_{b}} \sigma_{x} y d A=0 ; \\
y=-b: & \int_{A_{a}} \sigma_{y} d A=0 ; \\
y=-b: & \int_{\boldsymbol{A}_{a}} \sigma_{y} x d A=0 .
\end{array}
$$



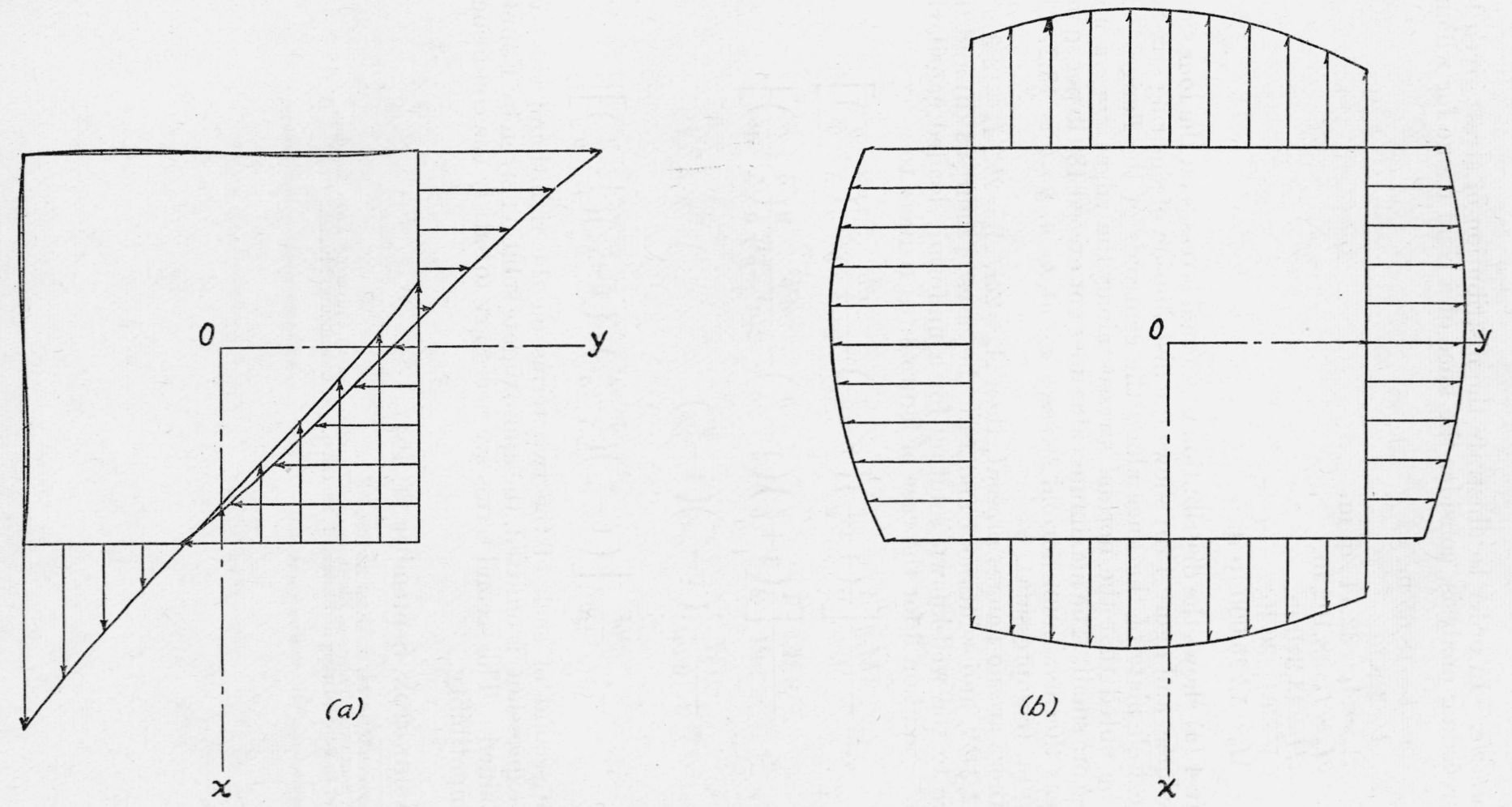
Example.-In order to illustrate the distribution of stress given by the preceding analysis, consider the knee of a rigid frame for which ${ }^{2}$

$$
\begin{aligned}
a & =b=18.65 \mathrm{in} . \\
t & =3 / 8 \mathrm{in} . \\
A_{a} & =A_{b}=32.44 \mathrm{sq}_{.} \text {in. } \\
I_{a} & =I_{b}=8,102 \mathrm{in}^{4} \\
H & =43,310 \mathrm{lb} . \\
V & =61,230 \mathrm{lb} . \\
M_{0} & =5,725,000 \mathrm{lb} \text { in. }
\end{aligned}
$$

Figure 4 (a) shows the distribution of normal stresses on the four sides of the knee, and figure 4 (b) shows the distribution of shearing stresses in the web plate of the knee along the centroids of the flanges. It may be noted that the normal stresses along the edges $x=-a$ and $y=-b$ are small. The maximum value does not exceed $180 \mathrm{lb}$ per sq in., whereas the maximum normal stress, $-\sigma_{x}$, at $x=a, y=b$ is $13,270 \mathrm{lb}$ per sq in. (compression).

If there are no flanges present, then $A_{a}=2 a t, A_{b}=2 b t, I_{a}=(2 / 3) a^{3} t$, $I_{b}=(2 / 3) b^{3} t$, and solutions 1 and 2 for the cases of figures $3(\mathrm{~b})$ and (c) reduce to the well-known solution for a uniformly loaded cantilever beam. ${ }^{3}$ Solution 3 for the case of figure $3(\mathrm{~d})$ reduces to

$$
\begin{aligned}
\sigma_{x}= & -\frac{3 M_{0} y}{2 b^{3} t}\left[\frac{1}{2}\left(1+\frac{x}{a}\right)^{2}\left(1-\frac{x}{2 a}\right)-\frac{a^{2} b^{2}}{2\left(a^{4}+b^{4}\right)} \frac{x}{a}\left(\frac{3}{5}-\frac{y^{2}}{b^{2}}\right)\right], \\
\sigma_{y}= & -\frac{3 M_{0} x}{2 a^{3} t}\left[\frac{1}{2}\left(1+\frac{y}{b}\right)^{2}\left(1-\frac{y}{2 b}\right)-\frac{a^{2} b^{2}}{2\left(a^{4}+b^{4}\right)} \frac{y}{b}\left(\frac{3}{5}-\frac{x^{2}}{a^{2}}\right)\right], \\
\tau_{x y}= & -\frac{9 M_{0}}{16 a b t}\left\{\left(1-\frac{x^{2}}{a^{2}}\right)\left(1-\frac{y^{2}}{b^{2}}\right)\right. \\
& \left.-\frac{a^{2} b^{2}}{3\left(a^{4}+b^{4}\right)}\left[\left(1-\frac{x^{2}}{a^{2}}\right)\left(\frac{1}{5}-\frac{x^{2}}{a^{2}}\right)+\left(1-\frac{y^{2}}{b^{2}}\right)\left(\frac{1}{5}-\frac{y^{2}}{b^{2}}\right)\right]\right\} .
\end{aligned}
$$

If the second of each of the two terms on the right-hand sides of these expressions is omitted, the approximate solution given by $\mathrm{Föppl}^{4}$ is obtained. The second terms are necessary to satisfy the conditions of compatibility.

Washington, September 11, 1941.

\footnotetext{
2 J. Research NBS 21, 269 (1938) RP1130.

3 R. V. Southwell, Theory of Elasticity, p. 377 (1936). Oxford University Press, London.

4 A. and L. Föppl, Drang und Zwang 1, 340 (1924). R. Oldenbourg, Munich.
} 